\title{
Effect of the environmental factors on diketopiperazine cyclo(Pro-Phe) production and antifungal activity of Bacillus amyloliquefaciens Q-426
}

\author{
Huiying $\mathrm{Yu}^{1} \cdot$ Jianhua Wang ${ }^{1,2} \cdot \mathrm{Xin}_{\mathrm{Li}^{1}} \cdot$ Chunshan Quan ${ }^{3}$ \\ Received: 31 October 2020 / Accepted: 18 February 2021 \\ (C) Institute of Molecular Biology, Slovak Academy of Sciences 2021
}

\begin{abstract}
Bacillus amyloliquefaciens Q-426 with strong antifungal activity was isolated from the compost samples in Dalian of China. Four kinds of diketopiperazines were extracted from the strain including cyclo(Pro-Phe) (cPP), which had cyclo(L-Pro-L-Phe), cyclo(D-Phe-L-Pro), cyclo(D-Pro-D-Phe) and cyclo(L-Pro-D-Phe) isomers. Results showed that (1) cPP production reached the maximum at $12 \mathrm{~h}$ and then maintained the constant yield; (2) cPP maintained a stable production in a wide temperature range of $31-42^{\circ} \mathrm{C}$; (3) cPP production were quite different for various carbon sources because of the different growth rate of the strain; (4) cPP maintained a higher level yield in neutral and weakly alkaline environment $(\mathrm{pH} 6-10)$ than acid $(\mathrm{pH} 3-5)$ environment. Moreover, cPP played a negative effect on the synthesis of antifungal substance when the bacteria maintained a good growth, and the previous studies found that cPP showed a positive response to biosensors which were used to detect signal molecules. Based on these studies, diketopiperazines were suspected as the signal molecule of B. amyloliquefaciens Q- 426 .
\end{abstract}

Keywords Diketopiperazines $\cdot \mathrm{cPP} \cdot$ Antifungal activity $\cdot$ Signal molecule

\section{Introduction}

Many bacteria isolated from nature, had been proven to be a rich source of diverse arrays of bioactive metabolites with great potential for pharmaceutical and medical applications. Bacillus amyloliquefaciens was discovered in soil by Fukumoto (1943), who named the bacterium because of it producing a liquifying amylase. In 1987 a group of scientists including Priest et al. (1987) established it as a separate species. B. amyloliquefaciens was a species of Gram-positive

Jianhua Wang

jhwang2014@163.com

1 Life Science College, Yuncheng University, Yuncheng 044000, People's Republic of China

2 Key Laboratory of Coastal Environmental Processes and Ecological Remediation, Yantai Institute of Coastal Zone Research, Chinese Academy of Sciences, Yantai 264003, People's Republic of China

3 Life Science College, Dalian Nationalities University, Dalian 116600, People's Republic of China bacteria which could produce many structurally diverse antimicrobial compounds, such as iturins, surfactins, fengycins.

Cyclo(Pro-Phe) (cPP) was a kind of diketopiperazines which formed from two amino acids by cyclodehydration. The diketopiperazines had been found in various microorganisms including Bacillus sp (Trichman et al. 2004; Lu et al. 2009; Wang et al. 2013; Shaala et al. 2016). Diketopiperazines (including cPP) and their derivatives were found to have a wide range of biological functions, such as antiviral (Sinha et al. 2004), antibacterial (De Kievit and Iglewski 2000; Fdhila et al. 2003; Kozlovsky et al. 2003; Gomes et al. 2019; Jia et al. 2019), antifungal (Kumar et al. 2014), antitumour (Nicholson et al. 2006; Gomes et al. 2019), antialzheimer (Turkez et al. 2020) and anticonvulsant (Dawidowski and Turto 2014). With a growing awareness of the diversity and biological roles played by the diketopiperazines found in nature, more and more attention had been paid to these compounds. Diketopiperazines were suspected to be signal molecule of Pseudomonas aeruginosa (Holden et al. 1999) and Pseudomonas putida WCS358 (Degrassi et al. 2002). They both observed that diketopiperazines showed positive response to signal molecule biosensor Agrobacterium tumefaciens NT1 
(pDCI41E33) or Escherichia coli JM109 (pSB401). Diketopiperazines, such as cyclo(Pro-Leu), cyclo(Val-Val), cyclo(Phe-Val), cyclo(Phe-Leul) and cyclo(Pro-Phe) were found as crossing communication quorum sensing signals between Cronobacter sakazakii and Bacillus cereus (Bofinger et al. 2017).

B. amyloliquefaciens Q-426 was isolated from compost samples collected in Dalian of China based on its antifungal activities and identified according to morphological and biochemical characteristics and 16S rDNA sequence (deposited in NCBI with a GenBank accession NO. HM130462). In previous studies (Zhao et al. 2014; Wang et al. 2016), diketopiperazines and antifungal compounds like bacillomycin $\mathrm{D}$, fengycin $\mathrm{A}$ and $\mathrm{B}$ were found produced in B. amyloliquefaciens Q-426. Here we found that environmental factors played a great influence on cPP production and antifungal activity. There were some relative connections between cPP and antifungal activity, and it might be helpful for evaluating the role of diketopiperazines played in the strain B.amyloliquefaciens Q-426.

\section{Materials and methods}

\section{Microorganisms and culture conditions}

B. amyloliquefaciens Q-426 was preserved in the China Center for Type Culture Collection (CCTCC NO. M2010237). The strain was grown at $37^{\circ} \mathrm{C}$ with $200 \mathrm{rpm}$ in a growth medium $\mathrm{CA}$, which contains $(\mathrm{g} / \mathrm{L}): \mathrm{K}_{2} \mathrm{HPO}_{4}, 1.5$; $\mathrm{KH}_{2} \mathrm{PO}_{4}, 0.6 ; \mathrm{NH}_{4} \mathrm{Cl}, 2.0 ; \mathrm{MgSO}_{4} .7 \mathrm{H}_{2} \mathrm{O}, 0.5 ; \mathrm{Na}$-Citrate, 7.5 , yeast extract, 8.0, and the initial $\mathrm{pH}$ was 6.5-7.0.

Candida albicans CGMCC 2.538 was obtained from the China General Microbiological Culture Collection Center. The growth medium YPD for this strain have been described previously ( $\mathrm{Li}$ et al. 2008).

\section{Extraction and quantitative analysis of diketopiperazine CPP}

The extraction and quantitative analysis method of cPP from the culture supernatants of $B$. amyloliquefaciens Q-426 had been described previously (Wang et al. 2010). In Brief, supernatants $(20 \mathrm{~mL})$ from stationary-phase cultures of B. amyloliquefaciens Q-426 extracted with equal volume of acidified ethyl acetate. The extract was removed by rotary evaporation $\left(40 \sim 45^{\circ} \mathrm{C}\right)$ and the residue resuspended in 1 $\mathrm{mL}$ acetonitrile. The crude extracts containing cPP were stored at $-20^{\circ} \mathrm{C}$ prior to analysis.

Quantitative analysis of cPP in the extract was performed by gas chromatography coupled with electron-impact mass spectrometry on GCMS-QP 2010 Plus (Shimadzu Corporation, Kyoto, Japan), and the detailed parameters set were described previously (Wang et al. 2010). The cPP produced by $B$. amyloliquefaciens Q-426 under different conditions were quantified basing on the peak area in total ion chromatogram (TIC) of GC-MS (gas chromatography-mass spectrometry).

\section{Extraction of antifungal substance and antifungal assays}

The extraction of antifungal substance from $B$. amyloliquefaciens Q-426 was based on the method described elsewhere (Wang et al. 2010). After concentrating and coprecipitating of the culture supernatant, the resulting pellet was redissolved in phosphate buffer solution (PBS). About $1 \mathrm{~mL}$ antifungal substance was obtained from $20 \mathrm{~mL}$ culture supernatant.

The antifungal activity of the samples was measured by the rate of inhibition on C. albicans, and $1 \mathrm{~mL}$ antifungal substance extract was added to $5 \mathrm{~mL} C$. albicans culture medium. A negative control test without antifungal substance was prepared at the same time. The inhibition rate of the antifungal substance was calculated by the following formula (Wang et al. 2010):

$\%$ inhibitionrate

$$
=\frac{\mathrm{OD}_{600} \text { ofControl }-\mathrm{OD}_{600} \text { of C.albicans Treated }}{\mathrm{OD}_{600} \text { ofBlank }} \times 100
$$

Here $\mathrm{OD}_{600}$ is the optical density at $600 \mathrm{~nm}$ of the sample examined. All of the results were quantified at least 5 times.

\section{The environmental factors investigated}

Diketopiperazine cPP yields were compared under different conditions, including variation of incubation time, temperature, $\mathrm{pH}$ level and carbon source in the media. The incubation time varied between $0 \sim 72 \mathrm{~h}(0,6,12,24,48$ and $72 \mathrm{~h})$. The temperatures investigated were $25,31,37,43$ and $49^{\circ} \mathrm{C}$. The $\mathrm{pH}$ levels were $3,4,5,7,8,9$ and 10 . The carbon sources used here were sodium citrate $(\mathrm{C})$, sodium acetate $(\mathrm{A})$, sodium formate $(F)$, glucose $(G)$, lactose $(L)$ and maltose $(M)$.

\section{Results and discussion}

\section{Characterization and quantification of CPP}

As already mentioned, the crude acetonitrile extract was detected by GC-MS, and mass analysis was performed in full-scan mode $(\mathrm{m} / \mathrm{z} 40-400)$. The GC-MS result of was shown in Fig. 1. In TIC shown in Fig. 1a, the peaks 1-8 were all for diketopiperazines (Wang et al. 2010, 2016), and peaks 7 and 8 correspond to cPP by comparision the EI-MS (electron ionization mass spectrometry) (Fig. 1c) with mass spectral library. The standard compound cyclo(L-Pro-L-Phe) gave the same retention time as peak 8 

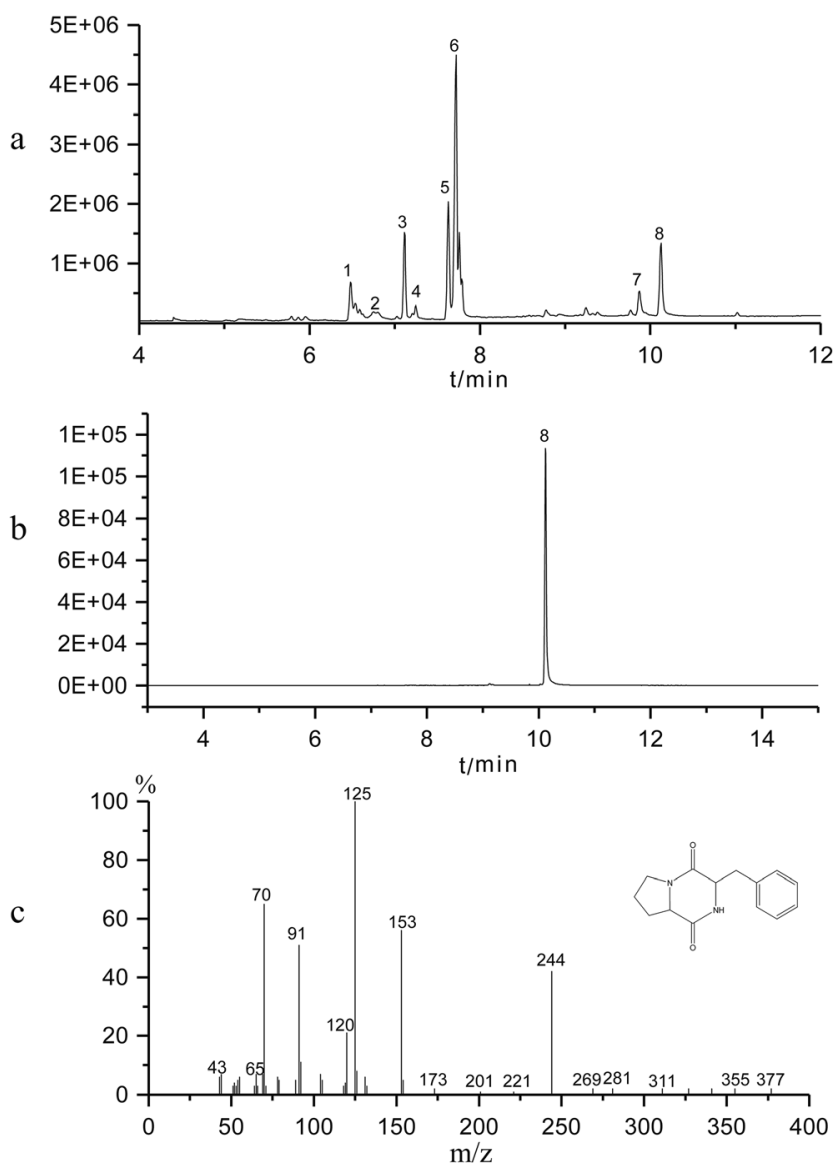

Fig. 1 GC-MS results of cPP extract. a Total ion chromatogram of the crude acetonitrile extract which contains 4 kinds of diketopiperazines: peaks 1 and 2 were for cyclo(Ala-Val), peaks 3 and 4 were for cyclo(Pro-Val), peaks 5 and 6 were for cyclo(Pro-Leu), peaks 7 and 8 were for cyclo(Pro-Phe) (cPP). b Total ion chromatogram of the standard compound cyclo(L-Phe-L-Pro), and peak 8 was used for quantitative analysis of cPP. c EI mass spectra of cPP (for peak 8) detected in the extract

(Fig. 1b), so peak 8 was suspected to be cyclo(L-Pro-L-Phe). However the achiral chromatographic column used in this study could not discriminate enantiomers, and cyclo(L-Pro-L-Phe) and cyclo(D-Pro-D-Phe) gave the same retention time, so peak 8 was for cyclo(L-Pro-L-Phe), cyclo(D-Pro-D-Phe) or the mixture of the two. Peak 7 might be for cyclo(D-Phe-L-Pro), cyclo(L-ProD-Phe), or the mixture of two. In this study, cPP was quantified on the basis the area of peak 8 .

\section{cPP synthesis in B. amyloliquefaciens Q-426 is incu- bation time dependent}

To determine the pattern of cPP production in B. amyloliquefaciens Q-426, diketopiperazines were extracted from the culture at different growth stages and analyzed by GC-MS. The cPP production and antifungal activity variation pattern during growth was shown in Fig. 2. Yield of cPP increased with bacteria growth and reached the maximum in late exponential phase (about $12 \mathrm{~h}$ after inoculation) (Fig. 2), corresponding with the growth pattern of general signal molecule. The complex medium used to culture B. amyloliquefaciens Q-426 includes yeast extract, which could produce cyclic peptides by heat sterilization Skwierczynski and Connors 1993). A little of cPP was produced by the culture medium, however it was certain that the cPP formed by the medium was much less than the cPP produced by the bacteria. After the late exponential phase, the concentration of bacteria maintained and declined slowly (Fig. 2), and the cPP concentration reduced gradually until almost to zero.

The antifungal activity gradually improved to the maximum at $24 \mathrm{~h}$, and last for 24 hours, then decreased with the reduction of cell biomass (Fig. 2). It was indicated that antifungal substance are produced only after completion of the bacteria growth phase. The highest antifungal activity came after the yield maximum of cPP, which suggested that the antifungal activity was under the regulation of diketopiperazines.

\section{CPP maintains a stable production in a wide range of temperature}

In a wide temperature range of $31-42^{\circ} \mathrm{C}, \mathrm{B}$. amyloliquefaciens Q-426 had a stable yield of cPP, which was consistent with the biomass of the strain (Fig. 3). This result confirmed that the amount of cPP was in proportion with the bacterial biomass, and the CPP yield was not dependent upon temperature changes in a specific range. Amazingly, the antifungal activity of the culture extracts reduced with the temperature increased (Fig. 3), which was quietly different from the trends of the biomass. Two hypotheses might explain the decrease of antifungal activity in high temperature: (1) It might because that low temperature was conducive to the synthesis of antifungal substances. (2) In the high-temperature environment, B. amyloliquefaciens $\mathrm{Q}-426$ enters into stationary phase in advance, and then became to wither up. The bacteria could not maintain a good growth until $48 \mathrm{~h}$, and the synthesis of secondary metabolites stopped, so the antifungal activity detected at $48 \mathrm{~h}$ reduced.

\section{Influence of the carbon source on CPP production}

To investigate the carbon source influence on diketopiperazines synthesis and antifungal activity, we changed the sodium citrate in CA medium with sodium acetate, sodium formate, glucose, lactose and maltose. Figure 4 showed that supplied with these carbon sources, B. amyloliquefaciens Q-426 could grow well, which was 
Fig. 2 Effect of incubation time on (a) the cell growth, (b) the yield of cPP, and (c) the antifungal activity of B. amyloliquefaciens Q-426. The cPP and antifungal substance were extracted respectively at 12 , 24 hours after inoculation. The cell growth was characterized by the value of $\mathrm{OD}_{600}$, and $\mathrm{cPP}$ was quantified basing on the peak area in TIC of GC-MS

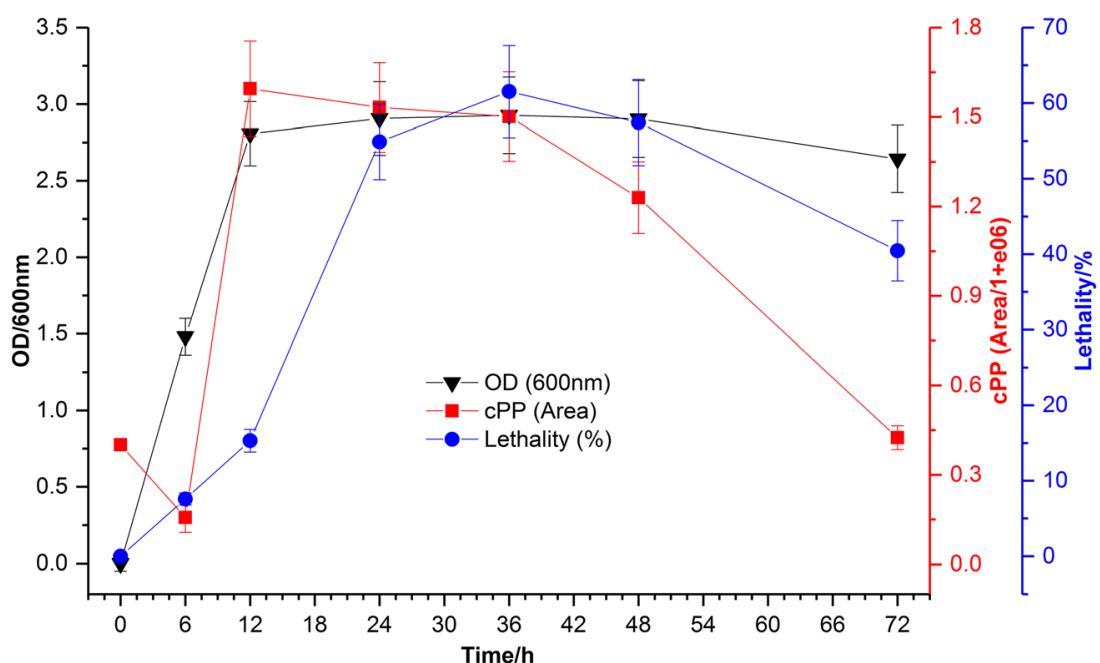

reflected in the values of $\mathrm{OD}_{600}$ (Fig. 4). However, the yields of cPP were quite different, cultured with glucose, lactose or maltose (sample G, L, M), B. amyloliquefaciens Q-426 produced more cPP than the others (sample C, A, F) at $12 \mathrm{~h}$ (Fig. 4). It might be because that the growth speed of the strain was different in mediums with different carbon sources, and the time went to late logarithmic phase and the bacteria biomass reached at stationary phase were quite different. B. amyloliquefaciens $\mathrm{Q}-426$ could produce antifungal substance at different culture medium, just with the different antifungal activity. Moreover, the antifungal activity was related to bacteria density and cPP production (Fig. 4). Cultured the strain with glucose, lactose or maltose (sample G, L, M), B. amyloliquefaciens Q-426 grows better and produced more $\mathrm{cPP}$, but the antifungal activity was lower. Compared with the sodium citrate and lactose mediums (sample C, L), we found that the same thing happen. It was indicated that the antifungal activity of the strain was not only related to the density of the bacteria, but also be inhibited by the diketopiperazines synthesis.

\section{Multiple effect of the medium pH on cPP production}

To investigate the $\mathrm{pH}$ influence on cPP synthesis, the yield of cPP was plotted as the function of $\mathrm{pH}$ levels (Fig. 5). Like most bacteria, B. amyloliquefaciens Q-426 grows well in the approximate neutral environment ( $\mathrm{pH}$ 6-9), other than strong acid or alkaline environment (Fig. 5). The strain could make a slight modification to the $\mathrm{pH}$ level of the initial culture medium (Fig. 6), and the $\mathrm{pH}$ level of the supernatant would be raised when the initial culture was neutral or acid, and be reduced when the initial culture was alkaline. As shown in Fig. 5, the cPP produced by $B$. amyloliquefaciens Q-426 was given priority to cyclo(L-Pro-L-Phe) or cyclo(D-Pro-D-Phe) in acidic and slight alkaline environment $(\mathrm{pH} \leq 8)$, and the other isomers (cyclo(DPhe-L-Pro), or cyclo(L-Pro-D-Phe)) in alkaline conditions ( $\mathrm{pH} \geq$ 9). Coincidentally, this phenomenon existed in other diketopiperazines, and it was indicated that the chirality of the diketopiperazines was affected by the acid-base property of the culture medium. In terms of total cPP, all the four isomers,
Fig. 3 Effects of incubation temperature on the cell growth ( $-\nabla-)$, the yield of cPP $(--)$, and the antifungal activity of B. amyloliquefaciens Q-426( $\rightarrow-)$

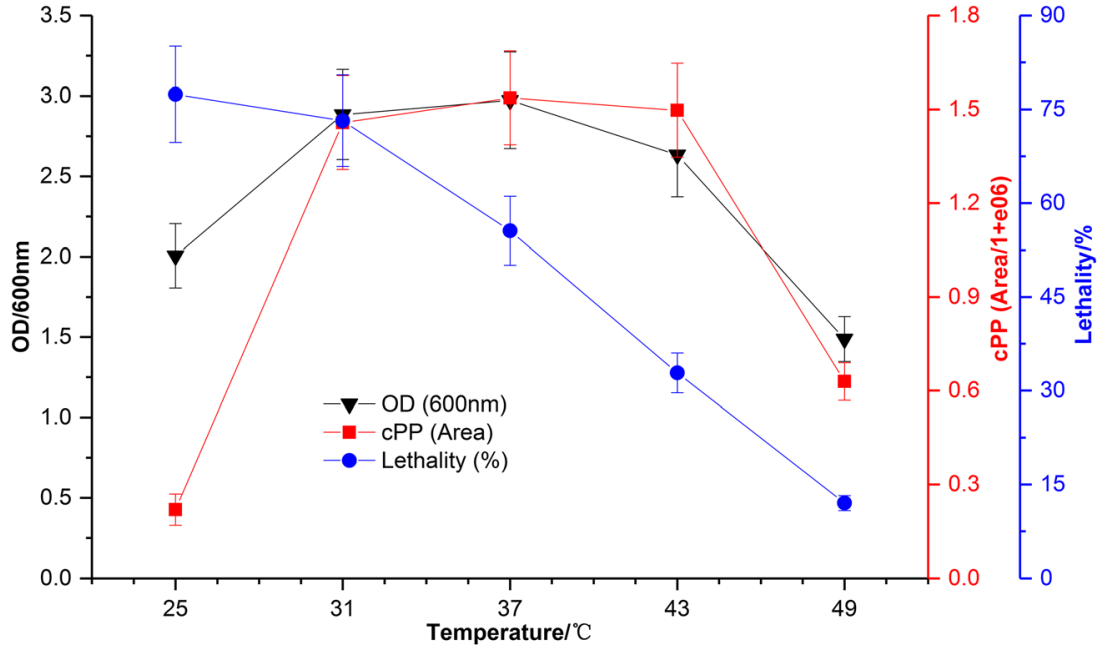


Fig. 4 Effects of carbon sources in the culture medium on the cell growth $(-\mathbf{v}-)$, the yield of cPP ( $--)$, and the antifungal activity of B. amyloliquefaciens Q-426 ( $--)$

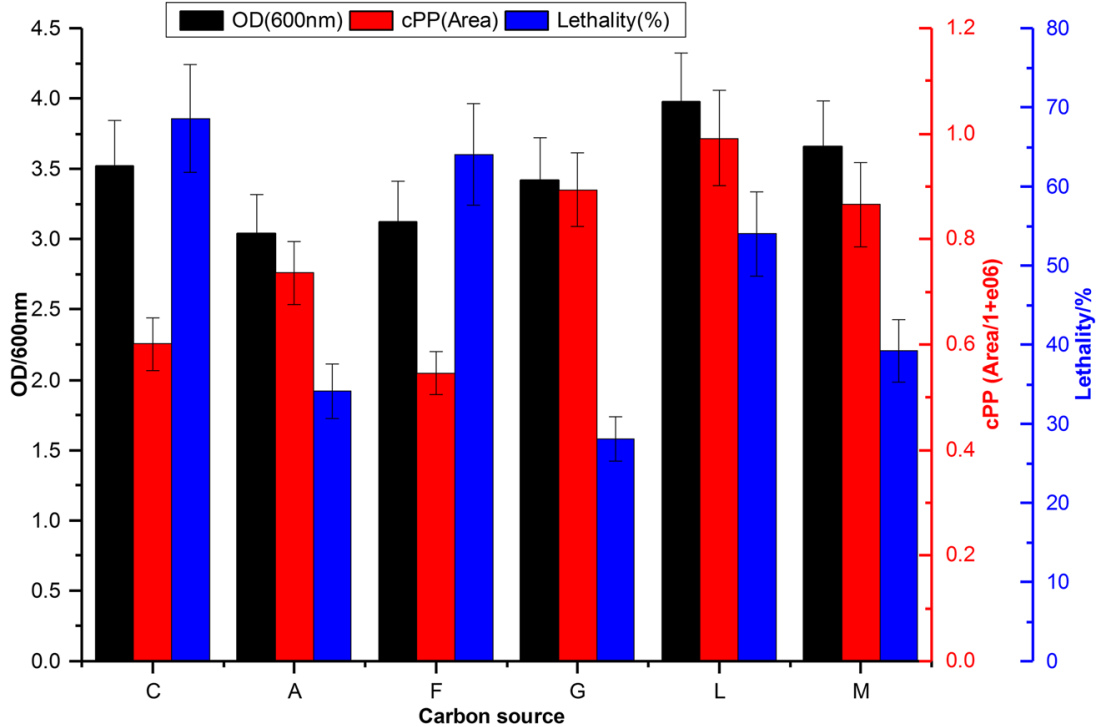

cultured in neutral medium, the strain could produce the most cPP, and then more in alkaline than acid medium. The Fig. 5 showed that neutral environment was the most suitable for antifungal substances synthesis, and with the $\mathrm{pH}$ increase or declining, antifungal activity were both decreased. Contrast the strain biomass, cPP yield, antifungal activity produced in weakly acidic $(\mathrm{pH}=6)$ and alkaline $(\mathrm{pH}=8)$ environment, we found that the weakly alkaline environment was more conducive to bacteria growth and cPP synthesis, while antifungal activity dropped compared with weakly acidic environment. It suggested that diketopiperazines might play a negative regulation role in antifungal substances synthesis.

\section{The role of cPP played in B. amyloliquefaciens Q-426}

Four kinds of diketopiperazines were found in the supernatant of B. amyloliquefaciens Q-426 (Wang et al. 2016), and as we discussed previously (Wang et al. 2010), diketopiperazines showed positive response to biosensors which used to detect AHLs (N-acyl-homoserine lactones). Previous studies on this compound showed that diketopiperazines had been suspected to be the signal molecule of $P$. aeruginosa (Holden et al. 1999) and $P$. putida (Degrassi et al. 2002). Studies in this paper suggested that diketopiperazine cPP might have some influence on the antifungal activity of the strain, and cPP were suspected to be the signal molecule of B. amyloliquefaciens Q-426.

As other organism behaviors, cPP production and antifungal activity were processes apparently dependent on many environmental factors, such as incubation time, temperature, carbon source and $\mathrm{pH}$. Research on the relationship of the cPP production and the antifungal activity of $B$. amyloliquefaciens Q-426 cultured in different environment would help us further understand the mechanism of action of diketopiperazine in the strain.
Fig. 5 Multiple effects of initial $\mathrm{pH}$ in the culture medium on the cell growth $(-\nabla-)$, the yield of enantiomers cyclo(L-Phe-L-Pro) and/or cyclo(D-Pro-D-Phe) (LLcPP, DD-cPP, $-\square-$ ), the yield of other enantiomers (cyclo(D-PheL-Pro), and/or cyclo(L-Pro-DPhe) (DL-cPP, LD-cPP, ---), and the antifungal activity of B. amyloliquefaciens Q-426 ( $\bullet-)$

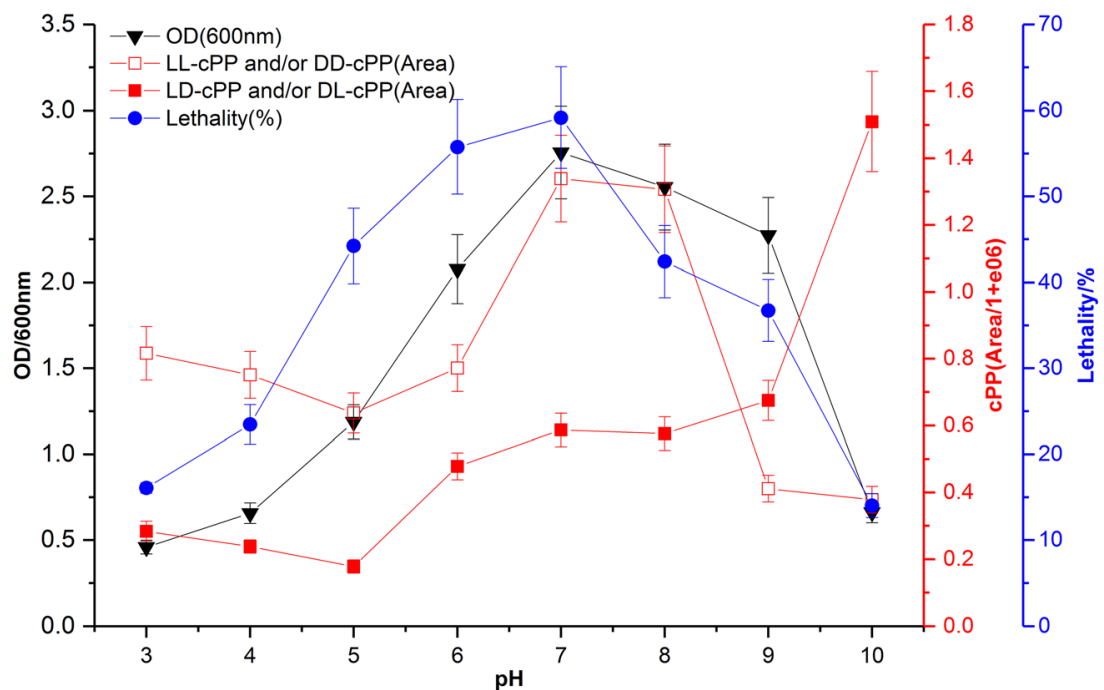


Fig. 6 The initial $\mathrm{pH}(-\bullet)$ in the culture medium and the final $\mathrm{pH}($ --) in the culture solution after incubated for 12 hours

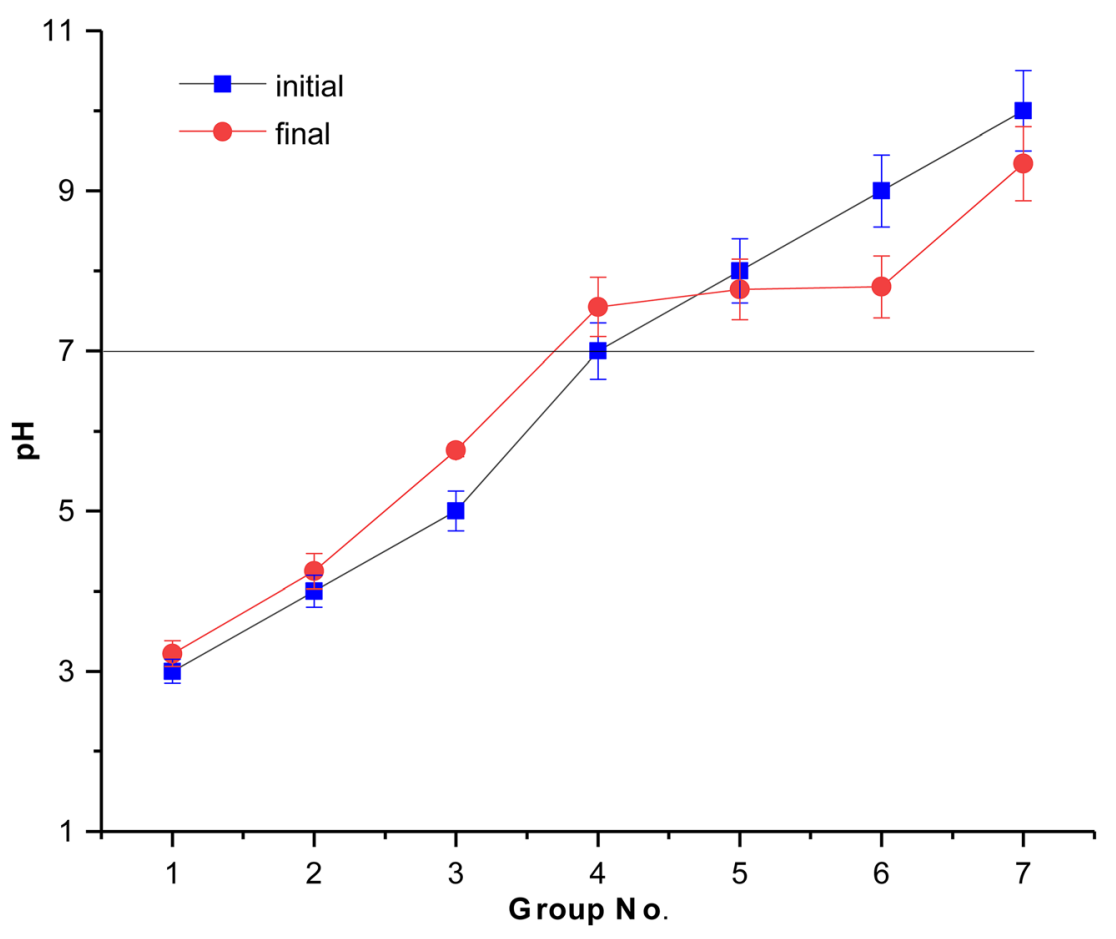

When the cells concentration reached the threshold, cPP was secreted to the medium gradually, and climbed to the maximum until the late exponential phase or the early stationary phase (Fig. 2). In other words, the concentration of cells and cPP climbed to the maximum at the same stage, proving that the production of cPP is a quorum sensing behavior. The hypothesis was supported by Fig. 3, which indicated that cPP yield and cell biomass maintains high in a wide temperature range of $31-42^{\circ} \mathrm{C}$. The result shown in Fig. 5 also indicated that the yield of cPP was directly related to the concentration of bacteria. The bacteria could consume cPP as carbon and nitrogen sources because of lacking of nutrition, so cPP almost depleted at $72 \mathrm{~h}$ (Fig. 2).

Variations in the fermentation environment factors often result in an alteration in antibiotics production. The alternation involves changes in both yield and composition of the compound (Upadhyay et al. 1991; Roitman et al. 1990) reported that by varying the conditions under which $B$. cepacia is grown, the yield and composition of the phenylpyrrole metabolites could be changed. Antibiotic produced by $B$. cepacia NB-1 also was greatly influenced by nutritional and environmental factors (El-Banna and Winkelmann 1998). Studies in this work indicated that the environmental factors might influence the yield of cPP and the antifungal activity of B. amyloliquefaciens Q-426. The Fig. 2 showed that the antifungal activity increased rapidly after the yield of diketopiperazines reached the maximum, which indicated that antifungal substance production was under the regulation of diketopiperazine. The results consisted with previous studies that the concentration of signal molecule reached a threshold can cause the expression of the gene which functioned the antibiotic production (Marahiel et al. 1993; Stein et al. 2002; Wang et al. 2014; Wu et al. 2017). Figure 4 showed that cPP played a negative effect on the synthesis of antifungal substance when the bacteria maintained a good growth, and it was supported by the results shown in Figs. 5 and 6. The present data clearly established that the antifungal activity of B. amyloliquefaciens Q-426 was not only influenced by environmental factors, but also was under the signaling regulation. Furthermore, the diketopiperazine cPP might act as signal molecule in the strain B. amyloliquefaciens Q-426.

Abbreviations cPP, cyclo(Pro-Phe); TIC, total ion chromatogram; PBS, phosphate buffer solution; GC-MS, gas chromatography-mass spectrometry; EI-MS, electron ionization mass spectrometry; AHLs, N-acylhomoserine lactones

Funding This work was supported by National Natural Science Foundation of China $(41,907,152)$, the Fund for Shanxi Key Subjects Construction (FSKSC) and Science and technology innovation project of Shanxi Universities (2019L0873).

\section{Declarations}

Conflict of interest The authors declare that they have no conflict of interest.

\section{References}

Bofinger MR, Sousa LS, Fontes JEN, Marsaioli AJ (2017) Diketopiperazines as cross-communication quorum-sensing signals 
between Cronobacter sakazakii and Bacillus cereus. ACS Omega 2: 1003-1008. https://doi.org/10.1021/acsomega.6b00513

Dawidowski M, Turto J (2014) Multicomponent synthesis and anticonvulsant activity of monocyclic 2,6-diketopiperazine derivatives. Med Chem Res 23:2007-2018. https://doi.org/10.1007/s00044013-0800-4

De Kievit TR, Iglewski BH (2000) Bacterial quorum sensing in pathogenic relationships. Infect Immun 68:4839-4849. https://doi.org/10. 1128/IAI.68.9.4839-4849.2000

Degrassi G, Aguilar C, Bosco M, Zahariev S, Pongor S, Ventui V (2002) Plant growth-promoting Pseudomonas putida WCS358 produces and secretes four cyclic dipeptides: cross-talk with quorum sensing bacteria sensors. Curr Microbiol 45:250-254. https://doi.org/10. 1007/s00284-002-3704-y

El-Banna N, Winkelmann G (1998) Pyrrolnitrin from Burkholderia cepacia: antibiotic activity against fungi and novel activities against streptomycetes. J Appl Microbiol 85:69-78. https://doi.org/10. 1046/j.1365-2672.1998.00473.x

Fdhila F, Vazquez V, Sanchez JL, Riguera R (2003) D, Ddiketopiperazines: Antibiotics active against Vibrio anguillarum isolated from marine bacteria associated with cultures of Pecten maximus. J Nat Prod 66:1299-1301. https://doi.org/10.1021/ np030233e

Fukumoto J (1943) Studies on the production of bacterial amylase I. Isolation of bacteria secreting potent amylases and their distribution (in Japanese). J Agric Chem Soc Japan 19:487-503

Gomes NGM, Pereira RB, Andrade PB, Valentao P (2019) Double the chemistry, double the fun: structural diversity and biological activity of marine-derived diketopiperazine dimers. Mar Drugs 17:551. https://doi.org/10.3390/md17100551

Holden MTG, Chhabra SR, Denys R, Stead P, Bainton NJ, Hill JP, Manefield M, Kumar N (1999) Quorum sensing cross talk: isolation and Chemical characterization of cyclic dipeptides from Pseudomonas aeruginosa and other Gram-negative bacteria. Mol Microbiol 33:1254-1266. https://doi.org/10.1046/j.1365-2958. 1999.01577.x

Jia B, Ma YM, Liu B, Chen P, Hu Y, Zhang R (2019) Synthesis, antimicrobial activity, structure-activity relationship, and molecular docking studies of indole diketopiperazine alkaloids. Front Chem 7:837. https://doi.org/10.3389/fchem.2019.00837

Kozlovsky AG, Zhelifonova VP, Adanin VM, Antipova TV, Ozerskaya SM, Ivanushkina NE, Grafe U (2003) Penicillium aurantiogriseum dierckx 1901: producer of diketopiperazine alkaloids (roqiefortine and 3, 12-Dihydroroquefortine), isolated from permafrost. Appl Biochem Microbiol 39:393-397. https://doi.org/10.1023/A: 1024572602458

Kumar SN, Sreekala SR, Chandrasekaran D, Nambisan B, Anto RJ (2014) Biocontrol of Aspergillus species on peanut kernels by antifungal diketopiperazine producing Bacillus cereus associated with entomopathogenic nematode. PLoS One 9:e106041. https://doi.org/ 10.1371/journal.pone.0106041

Li X, Quan C, Yu H, Fan S (2008) Multiple effects of a novel compound from Burkholderia cepacia against Candida albicans. FEMS Microbiol Lett 285:250-256. https://doi.org/10.1111/j.1574-6968. 2008.01238.X

Lu X, Shen Y, Zhu Y, Xu Q, Liu X, Ni X, Cao X, Zhang W, Jiao B (2009) Diketopiperazine constituents of marine Bacillus subtilis. Chem Nat Compd + 45:244-246. https://doi.org/10.1007/s10600009-9270-9

Marahiel MA, Nakano MM, Zuber P (1993) Regulation of peptide antibiotic production in Bacillus. Mol Microbiol 7(5):631-636. https:// doi.org/10.1111/j.1365-2958.1993.tb01154.x

Nicholson B, Lloyd GK, Miller BR, Palladino MA, Kiso Y, Hayashi Y, Neuteboom STC (2006) NPI-2358 is a tubulin-depolymerizing agent: in-vitro evidence for activity as a tumor vascular-disrupting agent. Anticancer Drugs 17:25-31. https://doi.org/10.1097/01.cad. 0000182745.01612.8a

Priest FG, Goodfellow M, Shute LA, Berkenley RCW (1987) Bacillus amyloliquefaciens sp. nov., nom. rev. Int J Syst Bacteriol 37:69-71. https://doi.org/10.1099/00207713-37-1-69

Roitman JN, Mahoney NE, Janisiewicz WJ (1990) Production and composition of phenylpyrrole metabolites produced by Pseudomonas cepacia. Appl Microbiol Biotech 34:381-386. https://doi.org/10. 1007/BF00170064

Shaala LA, Youssef DTA, Badr JM, Harakeh SM (2016) Bioactive $2(1 \mathrm{H})$-pyrazinones and diketopiperazine alkaloids from a tunicatederived actinomycete Streptomyces sp. Molecules 21:1116. https:// doi.org/10.3390/molecules21091116

Sinha S, Srivastava R, De Clercq E, Singh RK (2004) Synthesis and antiviral properties of arabino and ribonucleosides of 1,3dideazaadenine, 4-nitro-1,3-dideazapurine and diketopiperazine. Nucleos Nucleot Nucl 23:1815-1824. https://doi.org/10.1081/ $\mathrm{NCN}-200040614$

Skwierczynski RD, Connors KA (1993) Demethylation kinetics of aspartame and L-phenylalanine methyl-ester in aqueous-solution. Pharmaceut Res 10:1174-1180. https://doi.org/10.1023/A: 1018920402644

Stein T, Borchert S, Kiesau P, Heinzmann S, Kloss S, Klein C, Helfrich M, Entian KD (2002) Dual control of subtilin biosynthesis and immunity in Bacillus subtilis. Mol Microbiol 44:403-416. https://doi. org/10.1046/j.1365-2958.2002.02869.x

Trischman JA, Oeffner RE, de Luna MG, Kazaoka M (2004) Competitive induction and enhancement of indole and a diketopiperazine in marine bacteria. Mar Biotechnol 6:215-220. https://doi.org/10.1007/s10126-003-0010-z

Turkez H, Cacciatore I, Arslan ME, Fornasari E, Marinelli L, Stefano AD, Mardinoglu A (2020) Histidyl-Proline diketopiperazine isomers as multipotent anti-alzheimer drug candidates. Biomolecules 10:737. https://doi.org/10.3390/biom10050737

Upadhyay R, Visintin L, Jayaswal R (1991) Environmental factors affecting antagonisms of Pseudomonas cepacia against Trichoderma viride. Can J Microbiol 37:880-884. https://doi.org/10.1139/m91152

Wang J, Quan C, Qi X, Li X, Fan S (2010) Determination of diketopiperazines of Burkholderia cepacia CF-66 by gas chromatography-mass spectrometry. Anal Bioanal Chem 396: 1773-1779. https://doi.org/10.1007/s00216-009-3379-3

Wang P, Xi L, Liu P, Wang Y, Wang W, Huang Y, Zhu W (2013) Diketopiperazine derivatives from the marine-derived actinomycete Streptomyces sp. FXJ7.328. Mar Drugs 11:1035-1049. https://doi. org/10.3390/md11041035

Wang J, Zhang L, Teng K, Sun S, Sun Z, Zhong J (2014) Cerecidins, novel lantibiotics from Bacillus cereus with potent antimicrobial activity. Appl Environ Microbiol 80:2633-2643. https://doi.org/ 10.1128/AEM.03751-13

Wang J, Yang C, Fang S, Lu J, Quan C (2016) Inhibition of biofilm in Bacillus amyloliquefaciens Q-426 by diketopiperazies. World J Microbiol Biotechnol 32:143. https://doi.org/10.1007/s11274-0162106-4

Wu L, Guo X, Liu X, Yang H (2017) NprR-NprX quorum-sensing system regulates the algicidal activity of Bacillus sp. Strain S51107 against bloom-forming cyanobacterium Microcystis aeruginosa. Front Microbiol. 8:1968. https://doi.org/10.3389/fmicb.2017.01968

Zhao P, Quan C, Wang Y, Wang J, Fan S (2014) Bacillus amyloliquefaciens Q-426 as a potential biocontrol agent against Fusarium oxysporum f.sp. spinaciae. J Basic Microbiol 54:448 456. https://doi.org/10.1002/jobm.201200414

Publisher's note Springer Nature remains neutral with regard to jurisdictional claims in published maps and institutional affiliations. 